\title{
Erratum to: Treadmill Exercise Attenuates $\alpha$-Synuclein Levels by Promoting Mitochondrial Function and Autophagy Possibly via SIRT1 in the Chronic MPTP/P-Induced Mouse Model of Parkinson's Disease
}

\author{
Jung-Hoon Koo ${ }^{1,2}$ • Joon-Yong Cho ${ }^{1}$
}

Published online: 24 July 2017

(C) Springer Science+Business Media, LLC 2017

Erratum to: Neurotox Res

DOI 10.1007/s12640-017-9770-5

The original version of this article unfortunately contained mistakes and the authors hereby publishing these corrections. An error occurred in Fig. 8a during the final stages of figure mounting. The published bottom panel of Fig. 8a was inappropriately mounted with the wrong GAPDH Western blot control. The Fig. 8a should be corrected to remove and replace the GAPDH Western blot control from Fig. 8a. With these, the original article was corrected.

The corrected figure is presented on the next page.

The online version of the original article can be found at http://dx.doi.org/ 10.1007/s12640-017-9770-5

Joon-Yong Cho

chojy86@knsu.ac.kr

1 Department of Exercise Biochemistry, Korea National Sport University, Seoul 138-763, Republic of Korea

2 Institute of Sport Science, Korea National Sport University, Seoul 138-763, Republic of Korea 

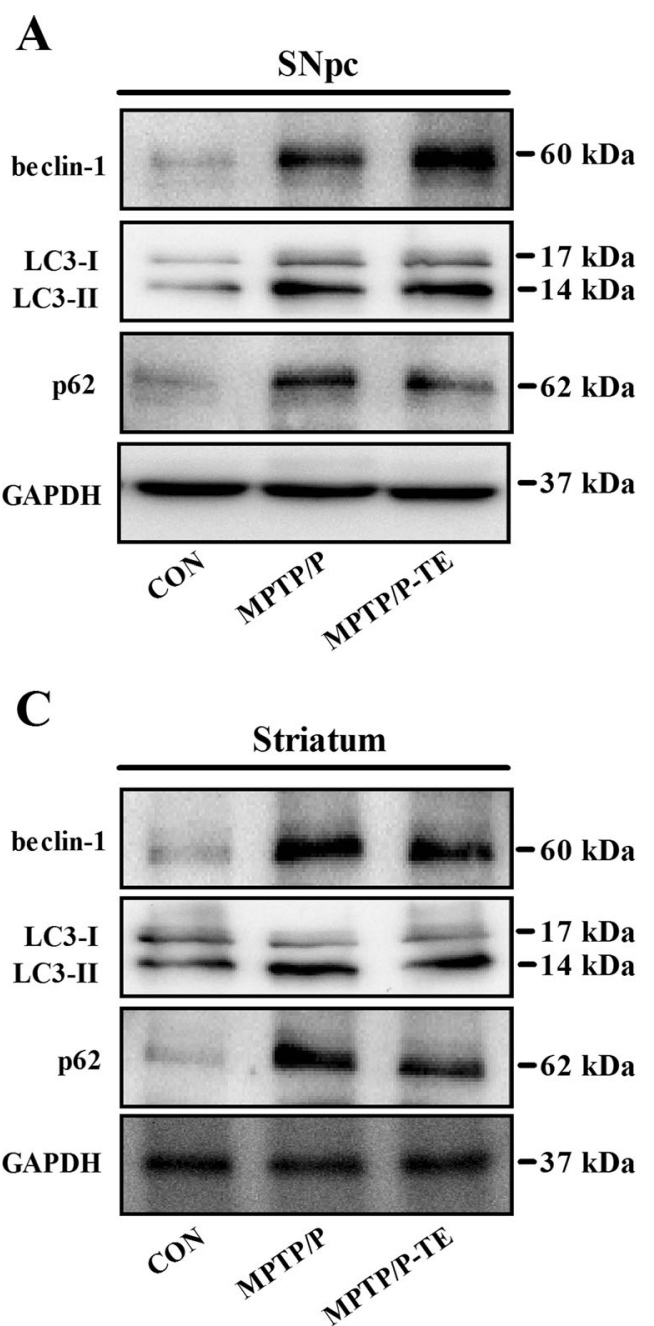

Fig. 8 Treadmill exercise promotes autophagy pathway in the MPTP/P mice model of PD. a Representative Western blot images of beclin-1, LC3, and p62 in the SNpc ( $n=5$ per group). b Quantification of beclin-1, LC3II, LC3II to LC3-I ratio, and p62 in the SNpc. c Representative Western blot images of beclin-1, LC3, and p62 in the striatum ( $n=5$ per group). d Quantification of beclin-1, LC3II, LC3II to LC3-I ratio, and p62 in the striatum. Values are expressed to $100 \%$ for
B

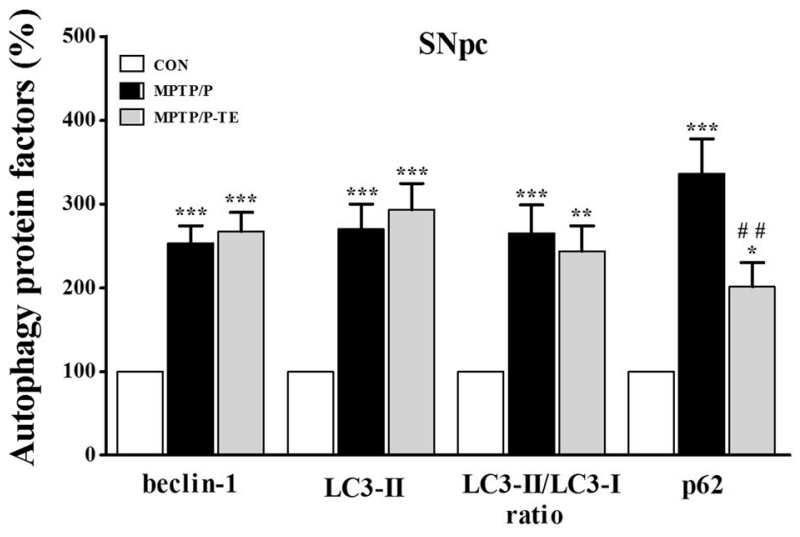

D

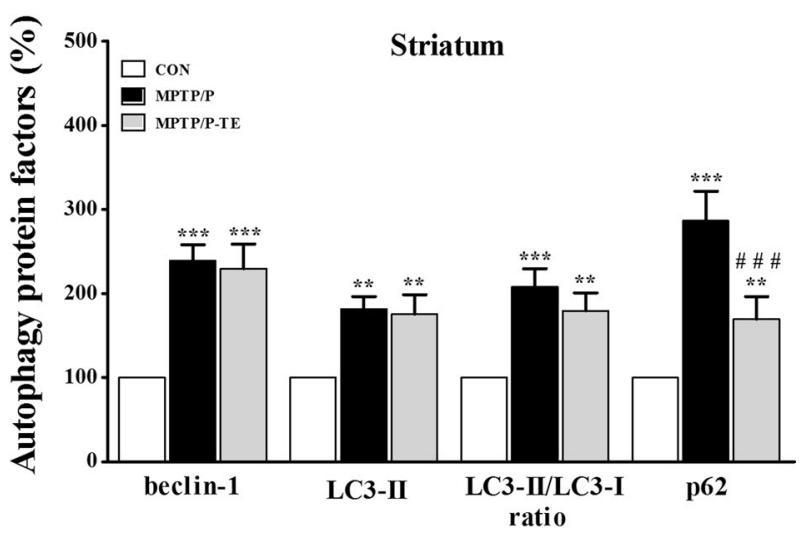

levels of the CON group. GAPDH was probed as housekeeping gene. Error bar represent as mean \pm standard error of mean (SEM). A Bonferroni post hoc test was conducted after the analysis of variance (ANOVA): $* p<0.05, * * p<0.01, * * * p<0.001$ versus the CON group; $\# \# p<0.01, \# \# p<0.001$ versus the MPTP/P group. Saline control $(\mathrm{CON}), \mathrm{MPTP}$ treatment with probenecid (MPTP/P), and MPTP treatment with probenecid plus treadmill exercise (MPTP/P-TE) 